\title{
Study on Modern Automotive Electronic Technology Characteristics and Development Trend
}

\author{
Zhufeng Chen \\ Hunan Vocational College of Modern Logistics, Changsha Hunan, 410131, China
}

Keywords: Modern automobile, Electronic technology, Characteristics, Trends.

\begin{abstract}
With the continuous development of information technology, the level of development of automobile industry for a country is largely based on the level of automotive electronic technology. To implement modern electronic technology and computer technology in the automotive industry has been rapidly applied; rapid development of modern automotive industry is the application of modern automobile electronic technology. Therefore, in this paper, the meaning of modern automobile electronic technology will be analyzed, the research of the applications field for modern automobile electronic technology, and generating characteristics and development trend of modern automobile electronic technology.
\end{abstract}

\section{Introduction}

Modern automobile development is primarily technical factors combined in the form of electronic information technology, mechanical technology and electronic products, information technology, and can improve the car's dynamic performance, handling performance and safety performance and other factors. Today, the car's advanced technology and electronic technology have achieved a unified model, across devices have been greatly utilized and created a broader space for development. So, in recent years, electronic technology has become an inevitable trend of modern automobile industry.

\section{Meaning of modern automobile electronic technology}

Modern automotive electronic technology in accordance with the development of electronic technology to build from the development of integrated circuit technology in the electronic device to the computer, not only to deepen the development of automotive electronics technology, also contributed to the steady progress of the automotive industry. They also improve the application and advancement of automotive electronics technology, automotive electronics technology development mainly in the functional implications of electronic control technology and the application of automotive electronics and information technology in two ways. Electronics and information technology in the automotive industry, mainly in the automotive environment, it is possible from the performance of the car itself, and independently-vehicle electronic devices. It is the use of electronic information technology, on-board computer systems are being developed, not only able to process the information, but also has speech recognition, navigation and image display. For functional electronic control technology, mainly based sensors, electronic control units and actuators composed it with the car itself can form close ties, to complete automatic control of the car itself. Such as: electronically controlled suspension, electronic fuel injection systems, etc. ${ }^{[1]}$. Currently, electronic devices in the car manufacturing has been more applications, in particular the installation of some limousine microcontroller, creating greater value and development power for vehicle development.

\section{The application of modern automobile electronic technology}

\section{Application on the chassis}

Modern automotive electronic technology in the chassis mainly in five areas, one for the electronically controlled automatic transmission, it can according to load, speed, vehicle speed and the state of the engine parameters, the use of computer calculations and determined that the shift the 
specific position of the lever, thus ensuring the transmission can be maintained within a reasonable time. The main advantage of this system is to achieve good acceleration effect, high sensitivity, and can produce accurate analysis traveling load, road conditions. Second, the anti-lock control system, which is mainly used in some of the more important security components. Able to control the car tires, car tires with the ground to ensure a reasonable rate slide, making the car in the road surface during braking, to ensure that the wheels and reasonable adhesion coefficient is formed between the ground, in order to reduce drag locking slide, unsafe factors can not only improve stability and safe operation of the car, but also to reduce the braking distance of a car. Third. For EPS, it is the use of a DC motor powered, this computer control system, compared with the traditional use of fewer parts, small size and relatively light weight. Optimization of steering effort, steering back to the positive features not only improve the car's steering ability, but also increase stability and maneuverability cars during car. Fourth, the self-adapting systems to timely regulation, to ensure the car during travel can achieve a certain stability and maneuverability. Fifth, the constant speed cruise control system, it is mainly used in high-speed long-distance, the driving resistance according to the throttle opening suspension system which according to the suspension of the load on the suspension stiffness of the spring, shock absorber damping characteristics progress automatically conditions and convenience for the driver to bring the vehicle speed during the adjustment.

\section{Application on the engine}

Automotive electronics technology in the engine, first, for the electronic control of fuel injection apparatus capable of automated operation, the output under certain power, to maximize fuel economy and clean air. According to the actual situation and the engine control law during the fueling operation, can change these laws compiled for the objective programs and stored in the computer storage. During engine carrying out its work, the use of sensors for air quality, exhaust oxygen content and temperature parameters were measured, and based on pre-programmed to implement the calculation, and then compared based on reasonable parameters, adjust the correct amount of oil . Secondly, electronic ignition devices, mainly based on computer, sensors, actuators and interfaces and other components ${ }^{[2]}$. It can detect the sensor according to the engine parameters analysis, judgment, and then the ignition is adjusted to save fuel and reduce air pollution. Moreover, the new engine electronic device can achieve automatic control, intelligent control and automation of diagnostic and other operations. Finally, intelligent variable valve timing technology, it enables the engine to achieve a reasonable proportion of the fuel, the engine speed under different circumstances, can achieve different fuel supply. Variable valve timing technology by the portion of the sensor, the controller and the ECU and other components, which can be adjusted according to the ignition timing, so as to achieve optimum operating conditions

\section{Characteristics of modern automobile electronic technology}

\section{Car security}

The use of modern automotive electronic technology to improve vehicle safety, the safety of the phenomenon mainly manifested as active and passive security control security control. Since the car active safety of electronic control systems installed, not only reduces driver fatigue, but also increase the safety of driving with a relaxed period. Active safety control system in order to avoid the main driver appears accidents and improve driver safety during driving. However, due to the response of electronic technology in the control process of the formation of relatively fast, it skilled, fast car operation, control, so during an emergency, can form a larger value in use. If a car accident, in order to reduce the collision of the car, to make use of traction control, anti-lock braking system to reduce the economic losses of the accident ${ }^{[3]}$. For example: collision prediction system, it can use radar, infrared obstacle detection, and control the running speed of the car, despite an emergency car phenomenon will occur prior alert to control the speed of the car.

\section{Control car}

Automobile brake control technology has been greatly developed, and to achieve more functionality. Automotive control, anti-lock control system for the development from the current drive skid system, 
traction system and electronic stability program systems. For a driver monitoring system, which can make a warning based on the behavior and reactions of the driver. For improved display system, it can use language to provide information to the driver, and the driver can conveniently keep driving carefully. For ESP, which prevents the car during emergency turns, sudden out of control, and reduce traffic accidents. Therefore, the use of modern automotive electronic control technology implementation, the reduction of the frequency of car accidents. For tire pressure monitoring system, tire pressure according to it, temperature detection of anomalies exist in the alarm and to prevent the occurrence of abnormal tire. Especially during car traveling on the highway, it is possible to reduce the accident to play a greater role, although vehicle collision, but also to protect the safety of the driver. Future implementations of intelligent systems have seat belts, airbags and collision prediction system is formed integrally, and to ensure the safety of the car with ${ }^{[4]}$.

\section{Car environmental protection and energy-saving}

With various industries around the world to achieve environmental protection and energy saving trend in the development of automobile industry development and the establishment of no exception. Many countries during manufacture automobiles are equipped with advanced engine management system, it can not only improve the operating efficiency of the engine, but also reduce the fuel consumption of materials and reduce atmospheric pollution. In order to enable the engine to achieve a good effect of fuel during operation, the use of advanced gasoline engine control system, not only the ignition timing of the engine, fuel injection for precise control, but also to control the variable intake air, VVT to improve the efficiency of the control change machines.

\section{The development trend of modern automobile electronic technology}

\section{Construction of vehicular electronics network system}

With the auto industry in the increasing number of electronic devices, the use of electronic devices to communicate with a major problem, build the vehicular electronics network system has become the main trend in the development of its automotive industry. Electronic apparatus can not only achieve a certain set of functions, but also for the service to other electronic devices, thereby enabling communication between electronic apparatus is formed as a vehicular electronics network system ${ }^{[5]}$. In this network system, not only make the car achieve comfort and safety during travel, but also improve the utilization efficiency of the electronic device. Construction of vehicular electronics network system but also on the line in the information received and transmitted can be analyzed according to the fault control problems existing in the system, and to identify the point of failure repair. Therefore, building a vehicular electronics network systems and providing technical basis for the safe driving of the vehicle.

\section{Green and environmental protection phenomenon}

As global warming continues to emerge, China has increased the importance of environmental protection. From the ever-increasing environmental awareness, people in the automotive industry but also for the environmental phenomenon made more demands, particularly for diesel engines, electronic control systems research and development and in-depth discussion, not only to focus on the electric power system and the hybrid system innovation and development, but also to achieve the green car, environmentally oriented development ${ }^{[6]}$. Fuel to power the car's development and implementation of innovative research is an important way to the development of green cars of the future. Automotive engine control, a reasonable allocation of the electronic system to improve vehicle operation economy and reduce vehicle emissions exists phenomenon. Today, China's auto industry development has achieved a new innovation and development, we began to use solar energy, bio-fuel and other automotive manufacturing environmental protection, but due to technical and financial problems, our trucks, heavy vehicles have to achieve the environmental protection of application form, China's auto industry in green development to achieve a new challenge.

\section{Energy}

China's automotive industry applications in electronic devices continues to increase, along with the energy use of the automotive industry, there are some problems. Such as: automotive power supply 
system voltage problems, make the driver can meet good driving form, will be analyzed according to the parameters during the design and manufacture of cars in existence. Our fuel prices have been increasing the efficiency of cars by people more attention. So, in this form, we should take advantage of the innovation and development of new technologies to promote energy development of China's automobile industry.

\section{Sensor Technology}

Sensor technology in China's auto industry has been greatly applied not only improve the level of China's automotive electronics industry, but also to enhance the level of automation in the automotive, electronic technology during automotive applications, to achieve a variety of functions and diversity development of the situation, so that the sensor technology to achieve new applications and development. Sensor technology enables the car to maintain stable operation, and improve the operating efficiency of automobiles ${ }^{[7]}$. Whether car during development or during travel, the sensor technology can be widely used, and therefore, during the sensor research and development, will have a lower cost of research and development, high safety and reliability of sensor technology, thus ensuring the sensor during application to form high precision. At present, China has achieved intelligent sensor technology, systematic and miniaturization of manufacturing and development. Car during driving, the most important is to ensure safety, car safety effect exists mainly in automobile driving two aspects, one is an active safety system, one for the passive safety systems. For active safety systems, mainly for accident prevention in the factors, and reduce the frequency of traffic accidents occur, such as: the use of ultrasonic sensors, optical sensors can not only achieve a certain degree of warning, but also to ensure safety during driving of automobiles sex. For passive safety systems, it is mainly applied during the accident, such as: seat belts, airbags and so are mature development.

\section{Mechatronics}

With the continuous development of science and technology, mechanical and electrical integration of applications in the automotive industry, mainly in the mechanical structure, and rapid development of automobile manufacturing. Mechatronics development not only reduce car emissions, but also reduce car pollution on the environment.

\section{Conclusion}

Modern automotive technology has been greatly developed, especially the application of electronic technology, the modern automotive industry formed a new impetus to the development. Modern cars use electronic technology can not only improve the power, economy and vehicle safety, but also to optimize the car's stability and comfort, and to achieve new developments in the field of automotive electronics technology development and application.

\section{References}

[1] Zhao Tongyu. Modern Automobile Electronic Technology Characteristics and Development Trend. Value Engineering, 2011,30 (6): 233-234.

[2] Chen Dongmei. Modern Automobile Electronic Technology Teaching Professional Mechanic College Student Comprehensive Quality Training. Science Mass (Science Education), 2015 (7): 136.

[3] Chen Wei. On the development and prospects of the modern automobile electronic technology. Science Review, 2015 (21): 229-229.

[4] Wang Na. Modern automobile electronic technology and its application in the instrument cluster. Science and Technology Information, 2013 (8): 114.

[5] Wang Rui Hong, Li Jing. Modern automotive electronics technology status and development trend analysis. Electronic world, 2014 (12): 16-16,17. 
[6] Jiao Liang. Modern automotive electronic technology application status. Electronic Design Technology, 2013, (10): 34-35.

[7] Jiang Feng, Zhu Enzhou. Exploration for the teaching methods of modern automotive electronics technology course. Science and Technology Vision, 2013 (4): 90. 